\title{
A Method for Parallel Arc Fault Detection and Identification
}

\author{
Yuan $\mathrm{Wu}$, Zhengxiang Song, Jianhua Wang \\ School of Electrical Engineering, Xi'an Jiaotong University \\ State Key Laboratory of Electrical Insulation \& Power Equipment \\ Xi'an, P.R.China \\ e-mail: jiemowang@126.com
}

\begin{abstract}
Every year, fires bring great loss and damage, and arc faults is one of main causes. But it is identified that the conventional circuit breakers fail to interrupt the circuit when an arc fault occurs. It is necessary to find new protecting techniques to improve the safety of circuits and reduce the probability of fires. This paper shows a simple method of detecting parallel arc faults. In this thesis, two significant characters of parallel arcs, the "shoulders" and the high amplitude, are obtained by experiments and then a circuit for discriminating the parallel arcs depending on them is designed. Other normal working household appliances, such as computers, air-conditions, are also have the similar characters, not easily distinguished from arc faults. The waveforms of those appliances are studied and compared with arcs. Results show that they still have different features in "shoulders" or amplitudes. At last, the correctness and reliability of this method are tested by an AFCI-tester.
\end{abstract}

Keywords-AFCI, parallel arc fault, detection and disrimination circuit

\section{INTRODUCTION}

More and more new electric equipments, such as computers, air-conditions, appear with the development of economy and industry. The safe of electric equipments become more and more important. But the incidence rates of electrical fires are still keeping high. A survey shows that home electrical problems account for 67,800 fires, 485 deaths, and $\$ 868$ million in property losses [1]. Many circuit interrupters do not work at all in fire accidents.

Studies show that it is possible to get the high temperature $\left(2000^{\circ} \mathrm{C} \sim 4000^{\circ} \mathrm{C}\right)$ only by $2 \mathrm{~A} \sim 10 \mathrm{~A}$ arc current. But conventional protecting techniques can't protect the circuits quickly when an arc fault occurs. In the Fig.1, it shows a typical time-current characteristic for a 20-A circuit breaker for over-current protection [2]. Actually, the curve is intended to protect conductors from thermal damage but not the effects of arcing. The shadowed area is out of the range of protection of the circuit breaker. The circuit breakers do not open the circuit in the conditions of shadowed area, while the arc fault may occur with great probability.

However, the energy from an arc in the region $1 \& 2$ is sufficient to cause ignition of flammable materials or organic insulation in such a short time. In the region 1 , the arc current may be formed line to neutral. We call it parallel arc. But in the region 2, the arc current is smaller, and it may occur in series with the load. We call it series arc. Fig.2 shows the differences of arcs.

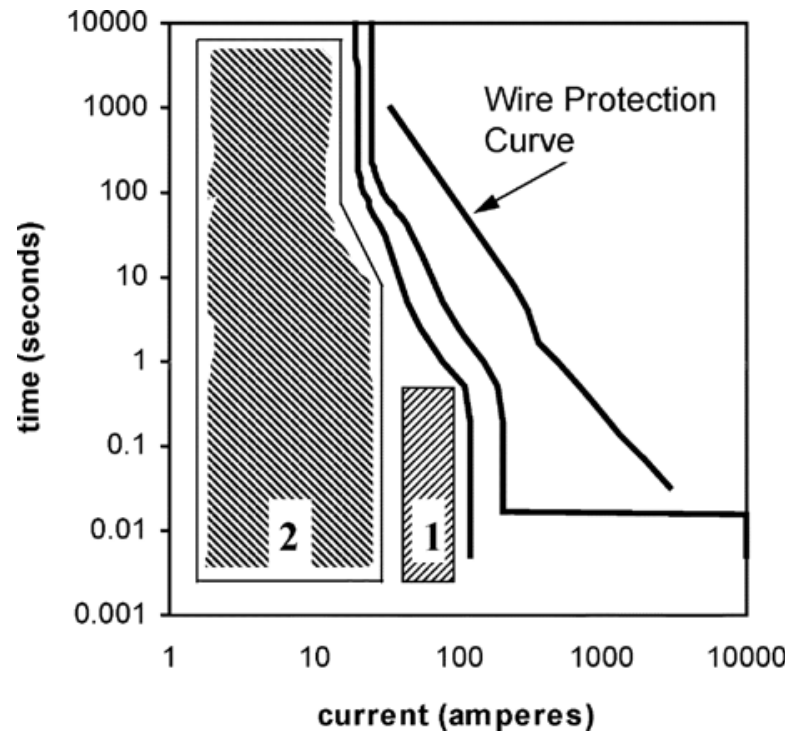

Figure 1. Time-current characteristic for a 20-A circuit breaker.

Uncontrolled arcing occurs in three modes: line-to-neutral (L-N or parallel), series, line-to ground (L-G), which are shown in the Fig.2 [3].

The parallel arc actually is a kind of short circuits and generally occurs at high level. A series arc is in series with a load and at lower level than the parallel arc. The L-G arc occurs when the arc reaches a ground path. This paper will focus on the parallel arcs and give a method to detect the parallel arcs.

In order to open the circuit and identify different arcs quickly and correctly, many researchers and corporations study the characters of arcs. A new circuit breaker called arc fault circuit interrupter (AFCI) is developed quickly. AFCIs can recognize the unique signatures of arcing faults and initiate a trip condition to isolate and de-energize the arcing fault.

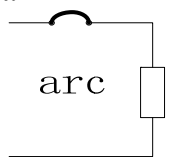

Series $\operatorname{arc}$

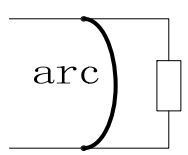

Paralle1

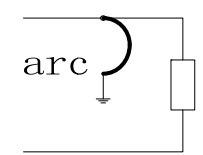

$\mathrm{L}-\mathrm{G} \operatorname{arc}$

Figure 2. Three different arcs modes based on the happening location.

Manufacturers on a National Electrical Manufacturers Association (NEMA) task force drafted an initial standard in 
the mid 1990s and worked with UL in the development of the final AFCI standard (UL 1699) which was published in Feb. 1999[4]. Now there are many mature AFCI products in the US. In China, the research of arc fault and AFCI is just started. So, it is significant and important to develop the AFCIs.

Researchers do lots of works to focus on the behavior and nature of arc faults. In general, two methods are used for detecting the arc. One is to measure the currents or voltages of arc and then analyze them by lots of different mathematical tools [5-9]. The other is to detect the signals of physics with an arc occurs [10-13].

In this paper, we will give the differences between the ordinary curve and the arc curve, and then we get the key points to distinguish the arc faults and the normal conditions. Two criterions are used. One is the amplitude of the current of the circuit; the other is the rate of changes when the current crosses the zero. A detection circuit is set up. We will get a significant pulse signal when the parallel arc occurs which is the control signal to cut the arc to protect the circuit quickly.

\section{THE ANALYSIS OF PARALLEL ARC CURRENTS}

AFCIs can distinguish the arc faults and normal conditions correctly and quickly. The key point is to find the unique signatures of arcing faults. So it is important and necessary to record different current curves of normal working equipments and the fault current curves when an arc fault occurs.

\section{A. The circuit and the structures of the experiments}

The structures of the experiments are prepared as Fig.3. The experimental setup was used to obtain parallel arcing voltage and current data for the $220 \mathrm{Vrms}, 50 \mathrm{~Hz}$ electrical system. The arc generator contains a moving electrode and a stationary electrode. We can create an arc by changing the distance between the two electrodes. The resistor is in series with the circuit to control the current. In order to obtain the magnitude of the current, a Hall current sensor is set up. The resistor will be replaced by other electrical equipment and the distance of the two electrodes is zero when we need to record the electrical equipment's working data.

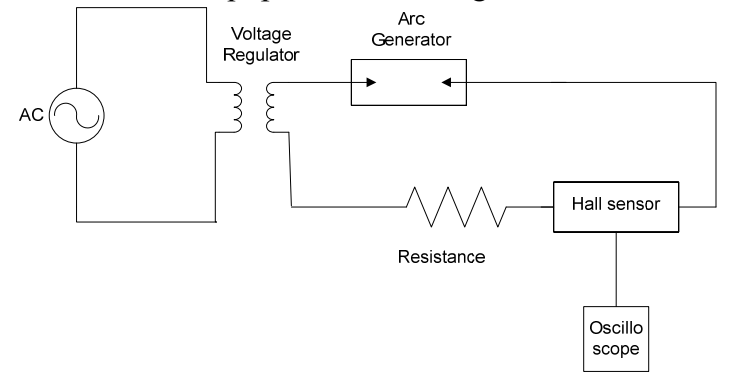

Figure 3. The structures of experimental setup.

B. The measurment of arc current and electrical equipments' current

In the Fig.3, the output of the Hall current sensor is proportional to the current of the circuit. They have the same phase, too. So, it is obviously for us to measure the voltage of the Hall sensor to get the magnitude of the current. We can get different levels of currents by changing the resistance. In the experiment, we changed the resistance three times. The resistance is $44 \Omega, 22 \Omega$ and $11 \Omega$ respectively. The material of the electrodes is copper. The curve of the current $(22.5 \mathrm{~A})$ when an arc occurs is shown as Fig.4. Other curves $(10 \mathrm{~A}, 5 \mathrm{~A})$ are similar with it. There is a zero-current section in each half cycle. We named these regions "shoulders". The rate of rise of arc current is usually greater than that for normal current, especially after the "shoulders". Besides, considering the parallel arc is actually a form of short circuit, the current has large amplitude.

Studies show that the curves of current of computers are similar to the curves of arcs. The computer's current is shown in Fig.5. The curve also contains the "shoulders" and the duration is long enough which is alike the arc. But the amplitude of computer's current is not so high.

Electric drill also has the similar characters which are shown as Fig.6. When the electric drill starts and works, we can see the high rate of rise of current. But the "shoulder" of the drill is not obvious and the duration time is shorter than the time of arcs. That is the biggest difference between them.

When an air-condition starts to work, the starting current may become an interference factor to detect an arc. The amplitude of air-condition's starting current is high, almost to $100 \mathrm{~A}$, and lasting time will reach to 10 cycles. The curve of the current is shown as Fig.7.
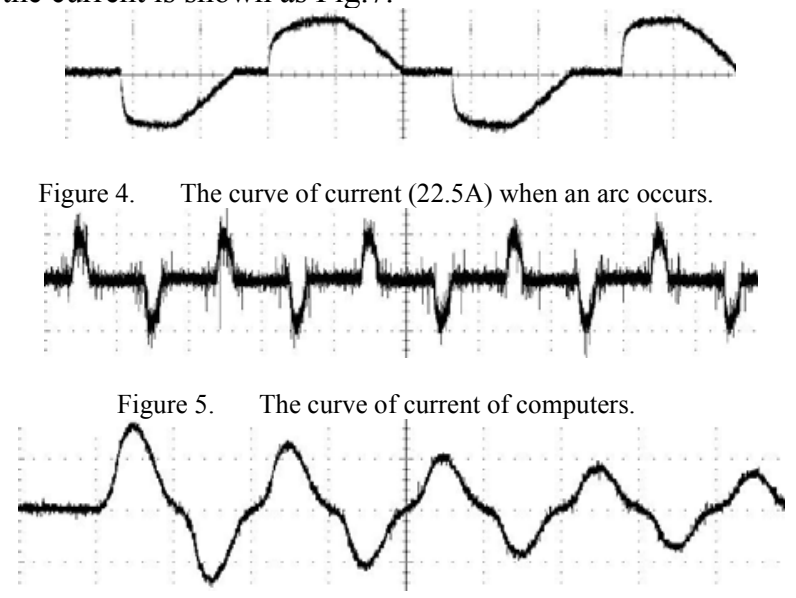

Figure 6. The curve of current of a drill.

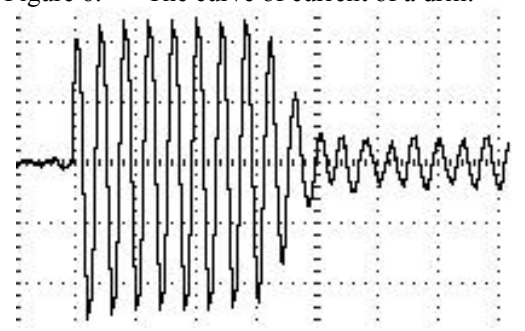

Figure 7. The curve of current of an air-condition. 


\section{The differences between the arcs and the equipments}

The two significant factors are "shoulders" and the high amplitude when an arc occurs. The curves of current of some equipment are similar to the arc, such as the computers and drills, while the amplitude of them is small. On the contrary, the current of air-conditions is very high but it do not contain the "shoulders". So, we get the two indispensable factors to confirm the arcs. One is the high amplitude and the other is the "shoulders".

\section{THE CRITERION OF THE PARALLEL ARCS}

\section{A. The introduction of the tester of AFCI}

The tester of AFCI is designed specially to test the correction and the rapidity of AFCIs. The tester can send out a pulse signal which lasts 8 cycles. The curve of the pulse signal is quick alike the arcs. So, in this experiment, we consider the pulse signal equal to the arc. In other words, if an AFCI can correctly open the circuit when we create a signal from the tester, we can confirm that the AFCI can correctly open the circuit when an arc occurs. The tester we used is produced by Ideal Suretest, and the amplitude of the current is 40A. The current of testers is shown as Fig.8.

\section{B. The design of cricuit to identify the arcs}

The main circuit is shown as Fig.9. In the experiment, we use the tester to produce the signal to imitate the arc signal, while we get the current by the Rogowski coil. The Hall sensor is used just for detecting the correction of the current. The arc detection circuit is shown as Fig.10. Two important criterions are used: the high amplitude and the high rate of rising of current. So the arc detection is separated by two parts. When the signal outputs from the Rogowski coil, it will pass an integrator, a low pass, a half-wave rectifier and a comparator to detect the amplitude of the current. At the mean time, it will also pass a high-pass filter, a window comparator, a charging capacitor and a comparator to detect the rate of the rising of the current. The two parts will be connected by an AND gate. Only the two important characters are fit meanwhile, the AND gate send out a signal which can be used for opening the circuit.

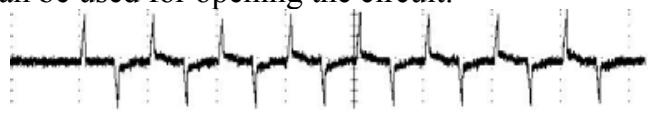

Figure 8. The current of an AFCI tester.

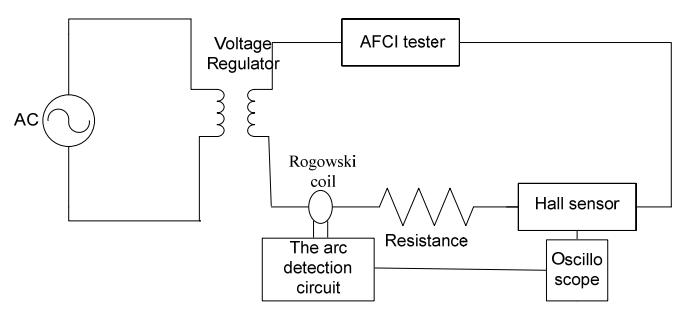

Figure 9. The block diagram of parallel arc detection.

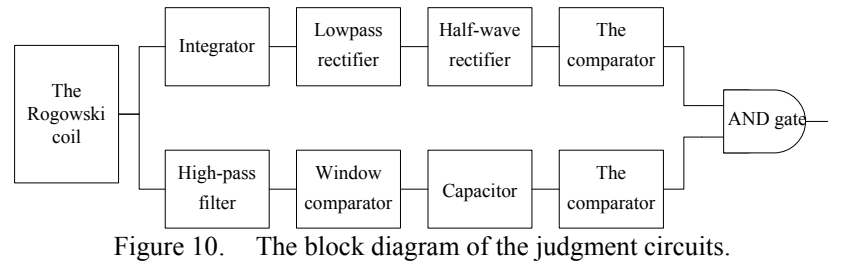

\section{The data of the detection circuit}

The following is the experiment to test the correct and the reliability of the detection circuit. The arc signal is imitated by an AFCI tester. The detection circuit contains two parts, one is the detection of the amplitude of current, the other is the detection of the high rate of rising when the current crosses zero.

1) The detection of the amplitude of current.

The data of the amplitude-detection module are shown as the Fig.11. Those signals are formed and recorded when an arc signal, outputting from the Rogowski coil, flows through the integrator, the low pass filter, the half-wave rectifier and the comparator orderly. The curve a is the output of the Hall sensor current when the AFCI tester sends out an arc signal. The curve $b$ is the output of the Rogowski coil and it passes through the integrator to become the current shown as the curve $c$. The curve $d$ is the output of low pass filter which is smoother than the curve c. The curve e is the output of the half-wave rectifier and the curve $f$ is the output of the comparator. The current of the curve $f$, which is a stable pulse signal, is the control signal to open the circuit when an arc occurs.

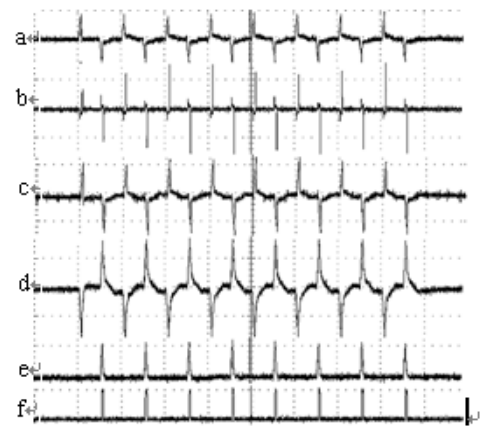

Figure 11. The waveforms of modules based on the amplitude judgment.

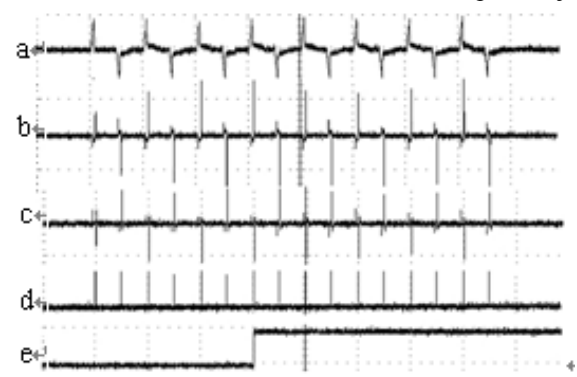

Figure 12. The waveforms of modules based on the rate-changing judgment.

2) The detection of the high rate of rising of shoulders. 
The detection of the rate of shoulders is mainly based on the differential action of the Rogowski coil. In this module, the current, outputting from Rogowski coil will pass through the high pass filter, the window comparator, the charging capacity, and the comparator. The data of the high rate of shoulders are shown as Fig.12. The curve a is the output of the Hall sensor current when the AFCI tester sends out an arc signal. The curve $b$ is the output of the Rogowski coil. Because of the differential effect of Rogowski coil and the high rates of change of voltage, the output contains such pulses. The curve $\mathrm{c}$ is the output of the high pass filter. Then the signal passes the charging capacity and the comparator to get curve d. In order to insure the correctness of this model, the signal is ordered to pass a capacitor to charging. When we got the signal in a time like curve e, we can confirm that, the number of pulses is not small. In other words, the appearance of pulses is not accidental. If the number of pulses or the charge of capacity is large enough, we can affirm the current in the circuit have the high rate of rising of shoulders.

\section{3) The detection of parallel arc fault.}

From the above, in order to confirm a parallel arc fault, we need two criterions, the detection of high amplitude and the detection of high rate of rising of shoulders. In this experiment, we use the AFCI tester to imitate the arc signal and set some definite parameters of each model considering the amplitude of the current of AFCI tester, the charging of capacity and so on. At last, the result is shown as Fig.13. The curve $a$ is the output of the Hall sensor. The curve $b$ is the detection signal of amplitude and the curve $\mathrm{c}$ is the detection signal of high rate of rising of shoulders. The curve $d$ is the final result of the AND gate to judge the arc signal.

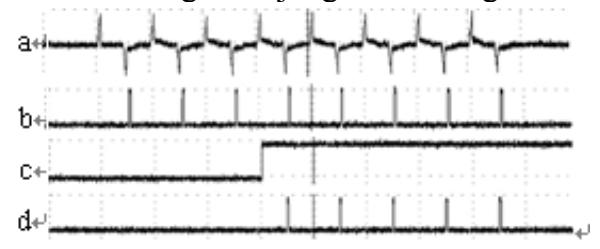

Fig. 1 The waveforms of modules based on the two judgment circuits.

\section{CONCLUSION}

In this paper, we design a system of distinguish the parallel arc fault based on the two significant characters, the high amplitude and the high rate of rising of shoulders, which the ordinary apparatus do not have when they are working regularly. The system can send out a high level signal when a parallel arc occurs, which can be used to open the circuit. We also use the AFCI tester to test and verify the correctness of the system and get the well result.

\section{ACKNOWLEDGMENT}

The authors would like to thank Mr. Xuandong Wang for devices providing and Dr. Xiaoshe Zhai for support on this study.

\section{REFERENCES}

[1] USFA.2006. On the Safety Circuit: A Factsheet on Home Electrical Fire Prevention. United States Fires Administration

[2] George G D, Wong Kon, Dvorak R F. More About Arc-Fault Circuit Interrupters [J]. IEEE Transactions on Industry Applications. 2004, 40(4): 1006-1011.

[3] UL Standard for Safety for Arc-Fault Circuit-Interrupters(UL 1699), April 7, 2006.

[4] J.J. Shea, Conditions for Series Arcing Phenomena in PVC Wiring, 51st IEEE Holm Conference on Electrical Contacts, pp. 167-175,Sept. 2005.

[5] Cheng Hong, Chen Xiaojuan, Xiao Wei, Wang Cong, Short-Time fourier Transform Based Analysis to Characterization of Series Arc Fault, PETIS 2009, 19-20 Dec.2009.

[6] Dongwei Li,Zhengxiang Song. A Method for Residential Series Arc Fault Detection and Identification. The Fifty-fifth IEEE Holm Conference on Electrical Contacts 7-12.

[7] Xin Zhou, Jerome Hastings, Thomas J. Schoepf. Application Feasibility of Detecting Glowing Contacts Using Acoustic Sensing Technology. The Fifty-fifth IEEE Holm Conference on Electrical Contacts 1-6. 